\title{
Chaotic Behavior in Piecewise Continuous Maps
}

\author{
Takahiro Yamaguchi and Hirokazu Ohtagaki \\ Dept. of Electrical and Electronics Engineering, Okayama University of Science \\ 1-1 Ridai-cho, Kita-ku, Okayama 700-0005, Japan \\ E-mail: ohtagaki@ee.ous.ac.jp
}

\begin{abstract}
In this paper, chaotic behavior in a piecewise continuous map(PCM) associated with sine circle maps of nonlinear systems of Van der Pol-Mathieu type is studied. At first, a sine circle map and its approximate $\mathrm{PCM}$ are derived.

Secondly, based on computational experiments on Lyapunov exponents to approximate PCM, existence conditions are shown to generate chaotic oscillation and periodic oscillation in PCM.

Finally, it is shown that the rotation numbers of oscillation in PCM form a part of successive Farey series and form devil's stairs in the region of cut off parameter such that Lyapunov exponent is positive. Furthermore, it is shown that the order of appearance of the oscillation in PCM has a self similar hierarchy.
\end{abstract}

\section{Introduction}

The importance of nonlinear dynamics has been considerably intensified by researchers $[1] \sim[3]$. Xin et al. and Liu et al. presented the chaos synchronization method to synchronize two identical chaotic systems with different initial values [2] [4]. Studies of nonlinear systems of Van der Pol-Mathieu type are important to design systems to be structurally stable $[5] \sim[8]$. In this paper, chaotic behavior of the oscillation in a piecewise continuous map $(\mathrm{PCM})$ associated with sine circle maps of the nonlinear systems of Van der PolMathieu type is studied. At first a sine circle map and an approximate PCM are derived, and based on Lyapunov exponents to approximate PCM, computational experiments are shown to generate chaotic oscillation and periodic oscillation in PCM. Secondary, we study chaotic behavior of the oscillation in PCM based on Lyapunov exponents to mapped points of PCM. The paper is organized as follows. In section 2, a sine circle map and an approximate PCM are derived, and based on Lyapunov exponents to approximate PCM, computational experiments are shown to generate chaotic oscillation and periodic oscillation in PCM. In section 3, it is shown that in the region of rotation number of oscillation in PCM form a subset of Farey series in region of the cut off parameter of PCM. In section 4 the order of appearance of the oscillation in the piecewise continuous map has a self similar hierarchy. In section
5 , concluding remarks of this paper are presented.

\section{Piecewise Continuous Map and Lyapunov Exponent}

In this section, chaotic behavior in piecewise continuous maps (PCM) associated with sine circle maps of the nonlinear systems of Van der Pol-Mathieu type is derived. Dynamics of the nonlinear system of Van der Pol-Mathieu type is written by the ordinary differential equation;

$$
\ddot{x}+\left(\beta-\delta x^{2}\right) \dot{x}+\left(1+\gamma x^{2}+\mu \cos (\omega \mathrm{t})\right) x=0,
$$

where $\beta$ denotes a dissipative coefficient, $\delta$ is a damping constant, $\gamma$ is a nonlinear parameter, $\mu$ and $\omega$ are an amplitude and an angular frequency of the periodic force. It is shown that the dynamical behavior in this nonlinear system exhibit chaotic in certain region of the damping parameter [25].

The angle variable to the system state is defined as

$$
\theta_{n}=\tan ^{-1}\left(\frac{x(n T)}{\dot{x}(n T)}\right), \quad n=0,1, \ldots
$$

where $T=2 \pi / \omega$. Sine circle map is obtained by a map;

$$
\theta_{n+1}=f\left(\theta_{n}\right), \quad n=0,1, \ldots
$$

By using root mean square method to the sine circle map, the approximate piecewise continuous map (PCM) can be expressed as

$$
\begin{gathered}
\theta_{n+1}=f_{1}\left(\theta_{n}\right):=A\left(\theta_{n}+C\right), \\
\left(0<\theta_{n}<\tilde{C}(=1 / A-C)\right), \\
\theta_{n+1}=f_{2}\left(\theta_{n}\right):=B\left(\theta_{n}-\tilde{C}\right), \\
\left(\tilde{C}<\theta_{n}<1\right) .
\end{gathered}
$$

Equation (3) is rewritten as a unified map;

$$
\boldsymbol{f}\left(\theta_{n}\right)=\left(\begin{array}{c}
f_{1}\left(\theta_{n}\right) \\
f_{2}\left(\theta_{n}\right)
\end{array}\right)
$$


where $A$ and $B$ are gradients of the maps $f_{1}$ and $f_{2}$, respectively, and $\tilde{C}$ is a cut off parameter of PCM defined by Eq.(4).

In the PCM, oscillation with $r$ times $m$ peaks $(r \cdot m)$ and $s$ times $(m+1)$ peaks $(s \cdot(m+1))$ is denoted as $\pi_{r, s}(m, m+1)$, where $r, s$ and $m$ are positive integers. Mixing degree $P$ and multiplicity $M$ in the oscillation $\pi_{r, s}(m, m+1)$ are defined as follows;

$$
\begin{aligned}
P & =r+s, \\
M & =m \cdot r+(m+1) \cdot s(=m \cdot P+s) .
\end{aligned}
$$

Rotation number $W$ of the oscillation $\pi_{r, s}(m, m+1)$ in the PCM can be defined as

$$
W=\frac{P}{M}=\frac{r+s}{m \cdot r+(m+1) \cdot s},
$$

(see Fig. 1).

In Fig. 1, $m$ periodic oscillation $\pi(m)$ is shown, where $\theta_{1}, \theta_{2}, \ldots, \theta_{m}\left(=\theta_{6}\right)$ indicate mapped points of the $\mathrm{PCM}$, and rotation number to a given initial value $\theta_{0}$ is given as

$$
W=\frac{P}{M}=\frac{1}{m} .
$$

Lyapunov exponent of the PCM is defined as

$$
\begin{gathered}
L=\frac{1}{M} \log B^{M-P} \log A^{P} \\
=\frac{1}{M}\left(M \log B\left(1-\frac{\frac{P}{M}}{\frac{|\log B|}{\log A}}\right)-P|\log B|\right) \\
=\left(1-\frac{\frac{P}{M}}{\frac{|\log B|}{\log A}}\right)\left(\frac{M-P}{M}\right)|\log B|,
\end{gathered}
$$

where $(M-P)$ and $P$ are positive. It is well known that if $L>0$, then the motion of the mapped points is chaotic, and that if $L<0$, then the motion of the mapped points is periodic or quasi periodic. Let the value of the cut off parameter $C$ such that $L=0$ be $C_{\mathrm{C}}$ that is called as a critical cut off parameter.

From the results of computational experiments, the following proposition is stated (see Fig. 2).

\section{Proposition 1 (Existence of Chaotic Behavior and Existence of Periodic Oscillation)}

If, for the critical cut off parameter $C_{\mathrm{C}}$, inequality

$$
C<C_{\mathrm{C}}
$$

holds, then the behavior in the PCM is chaotic. If, for the critical cut off parameter $C_{\mathrm{C}}$, inequality

$$
C_{\mathrm{C}}<C
$$

holds, then the oscillation in the PCM is periodic.

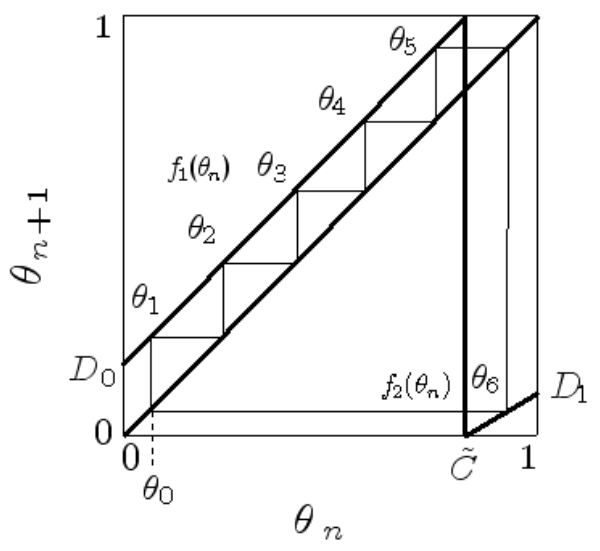

Fig. 1: Mapped Points in Approximate Piecewise Continuous Map $(m=6$.)

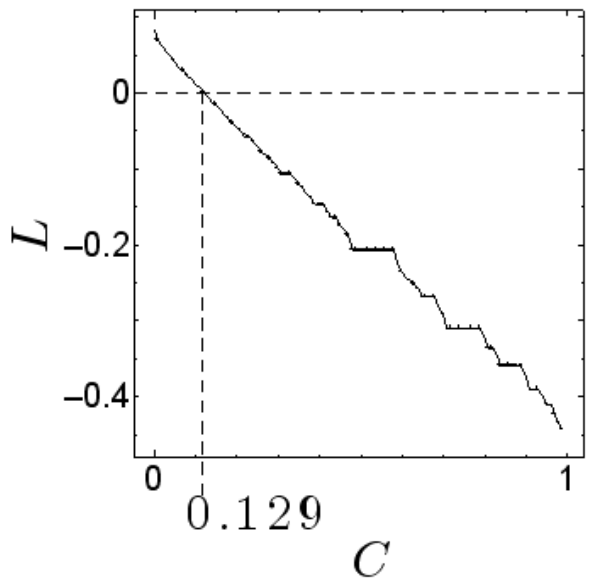

Fig. 2: Lyapunov Exponent in Approximate Piecewise Continuous Map $\left(C_{\mathrm{C}}=0.129\right.$. $)$

Equation (8) can be rewritten as:

$$
L=\frac{1}{M} \log B^{M-P}+\log A^{P} .
$$

Let $M=m_{0}, P=1$ for $L=0$. From Eq.(10), it follows that

$$
\begin{gathered}
L=\frac{1}{m_{0}}\left(\left(m_{0}-1\right) \log B+\log A\right)=0, \\
m_{0}=1-\frac{\log A}{\log B} .
\end{gathered}
$$


Therefore, from Eq.(12), it follows that the corollary 1 holds.

\section{Corollary 1}

There exist periodic oscillations $\pi(m)$ for positive integer $m$ that satisfies the inequality

$$
m \leq M_{\max }=\left[m_{0}\right],
$$

where [.] denotes the part of maximum integer of the real number $(\cdot)$.

From corollary 1 and Eq.(12), it follows that the periodic oscillations $\pi(m)$ exist for $m$ such that $1 \leq m \leq$ $M_{\max }=6\left(<m_{0}=6.359\right)$ for $A=1.1$ and $B=0.6$, which are derived from the sine circle map associated with the van der Pol-Mathieu system.

\section{$3 \quad$ Rotation Number and Farey Series}

Various oscillations $\pi_{r, 1}(m, m+1)$ and the oscillations $\pi_{1, s}(m, m+1)$ are shown in Fig. 3 for certain regions of cut off parameter. It is shown that oscillations exist successively $\pi_{1,3}(4,5), \pi_{1,2}(4,5), \pi_{1,1}(4,5), \pi_{2,1}(4,5)$, $\pi_{3,1}(4,5)$, which correspond to rotation numbers in series; $4 / 19,3 / 14,2 / 9,3 / 13,4 / 17$ and form a part of a successive Farey series with denominator less than 19. The Farey series $F_{M}$ of order $M$ is the monotonically increasing sequence of all irreducible rationales between 0 and 1 whose denominators do not exceed $M$, thus $\frac{P_{i}}{M_{i}}$ belongs

$$
F_{M}=\frac{1}{5}, \frac{1}{4}, \frac{1}{3}, \frac{2}{5}, \frac{1}{2}, \frac{3}{5}, \frac{2}{3}, \frac{3}{4}, \frac{4}{5}, \frac{1}{1} .
$$

A Farey sequence has a property such that if $\frac{P_{i-1}}{M_{i-1}}$ and $\frac{P_{i}}{M_{i}}$ are consecutive terms of $F_{M}$, then

$$
P_{i} M_{i-1}-P_{i-1} M_{i}=1 .
$$

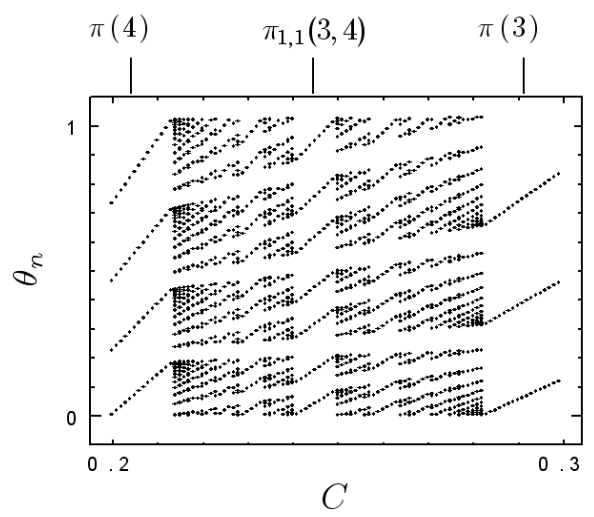

(a)

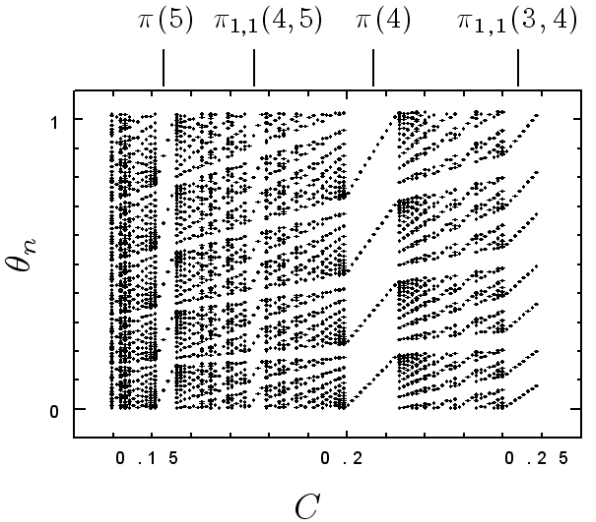

(b)

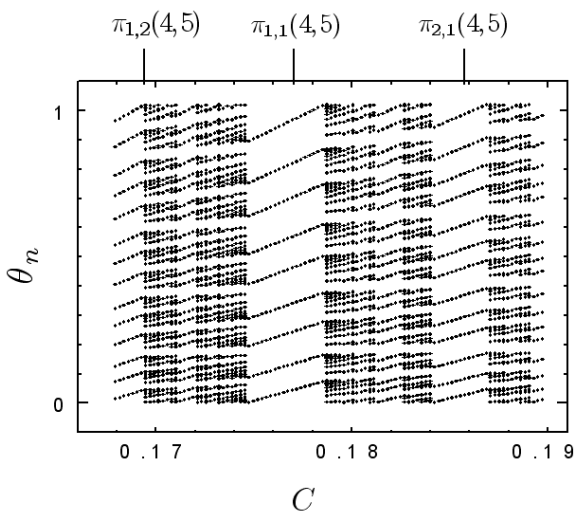

(C)

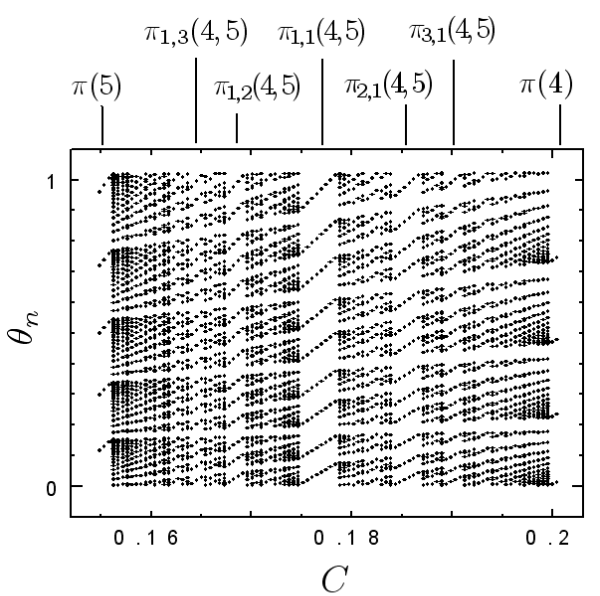

(d)

Fig. 3: Various oscillations $\pi_{r, s}(m, m+1)$ in PCM ((a): $C=0.2 \sim 0.3$, (b): $C=0.15 \sim 0.25$, (c): $C=0.17 \sim 0.19,(\mathrm{~d}): C=0.155 \sim 0.20$.)

In Fig.4, the rotation numbers are shown to a certain region of cut off parameter. From Fig.4, it can be seen that rotation numbers of oscillations in PCM form devil's stairs. 


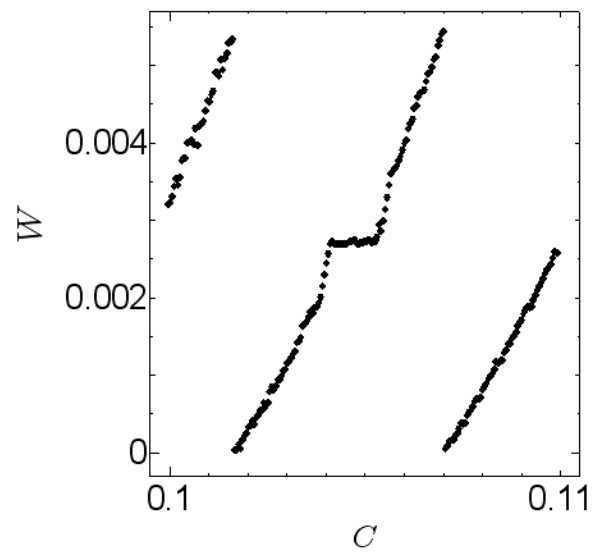

Fig. 4: Rotation Numbers vs Cut Off Parameter $C$

\section{Self Similar Hierarchical Structure}

The following equation:

$$
\begin{gathered}
\theta_{n+1}=a_{m} \theta_{n}+c_{m} C-B \\
=H\left(\theta_{n}\right)=f_{2}\left(f_{1}^{m}\left(\theta_{n}\right)\right), \\
\left(0<\theta_{n}<\frac{B-c_{m-1} C}{a_{m-1}}\right)
\end{gathered}
$$

holds for $(m+1)$-PO, where $m$-Peak Oscillation is abbreviated as $m$-PO, $f^{m}(\cdot)$ denotes iteration of $m$ times of the function $f_{1}(\cdot)$. The following equation:

$$
\begin{gathered}
\theta_{n+1}=a_{m-1} \theta_{n}+c_{m-1} C-B \\
=G\left(\theta_{n}\right)=f_{2}\left(f_{1}^{(m-1)}\left(\theta_{n}\right)\right) \\
\left(\frac{B-c_{m-1} C}{a_{m-1}}<\theta_{n}<\frac{a_{m} B-a_{m} a_{m} c_{m-1} C}{a_{m-1}}\right. \\
\left.+c_{m} C-B\right)
\end{gathered}
$$

holds for $(m+1)$-PO and $m$-PO, respectively, where

$$
a_{m}=A^{m} B, \quad c_{m}=A_{m} B, \quad A_{m}=\sum_{i=0}^{m} A^{i} .
$$

Let oscillation $K^{(2)}$-PO be oscillation consisting of $K$ times $(m+1)-\mathrm{PO}$ and $\mathrm{K}$ times $m$-PO. The following equations

$$
\begin{gathered}
\theta_{n+1}^{(2)}=a_{K}^{(2)} \theta_{n}^{(2)}+c_{K-1}^{(2)} C-B_{K-1} \\
=G\left(H^{(K)}\left(\theta_{n}\right)\right),
\end{gathered}
$$

$$
\begin{aligned}
\left(0<\theta_{n}^{(2)}<\frac{B_{K-2}-c_{K-2}^{(2)} C}{a_{K-1}^{(2)}}\right), & (17 a) \\
\theta_{n+1}^{(2)}= & a_{K-1}^{(2)} \theta_{n}^{(2)}+c_{K-2}^{(2)} C-B_{K-2} \\
= & G\left(H^{(K-1)}\left(\theta_{n}\right)\right), \\
\left(\frac{B_{K-2}-c_{K-2}^{(2)} C}{a_{K-1}^{(2)}}<\right. & \theta_{n}<\frac{a_{K}^{(2)} B_{K-2}-1_{K}^{(2)} c_{K}^{(2)} c_{K-2}^{(2)} C}{a_{K-1}^{(2)}} \\
& \left.+c_{K-1}^{(2)} C-B_{K}\right) . \\
a_{K}^{(2)}=\pi_{m}^{K} a_{m-1}, & (17 b) \\
c_{K-1}^{(2)} & =\sum_{i=0}^{K-1} a_{m}^{i} a_{m-1} c_{m} c_{m-1}, \\
B_{K-1} & =\sum_{i=0}^{K-1} a_{m}^{i} a_{m-1} B+B
\end{aligned}
$$

hold for $\left(K+1^{(2)}-\mathrm{PO}\right)$ and $\left(K^{(2)}-\mathrm{PO}\right)$, respectively.

Consider the region of cut off parameter that the oscillation $\pi_{1,1}(m, m+1)$ and oscillation $\pi(m+1)$ exist, and that the oscillation $\pi^{(2)}(K)$ such as

$$
\pi^{(2)}(K+1) \equiv \pi_{1, K}(m, m+1)
$$

exist. The inequality

$$
\frac{c_{K-1}^{(2)}}{B_{K-1}}<C<\frac{B_{K-1}+\left(a_{m}^{K} a_{m-2}+a_{K}^{(2)}\right) a_{0}}{a_{m}^{K-1} a_{m-1}^{2} a_{0}+a_{K-1}^{(2)} c_{m-1}+c_{K-1}^{(2)}}
$$

holds in the region of cut off parameter $C$ that $\pi^{(2)}(K+$ $1)=\pi_{1, K}(m, m+1)$ does exist. The inequality

$$
\frac{B_{K-1}+\left(a_{m}^{K} a_{m-2}+a_{K}^{(2)}\right) a_{0}}{a_{m}^{K-1} a_{m-1}^{2} a_{0}+a_{K-1}^{(2)} c_{m-1}+c_{K-1}^{(2)}}<C<\frac{c_{K-3}^{(2)}}{B_{K-3}}
$$

holds in the region of $C$ that $K^{(2)}-\mathrm{PO}$ exist. Making the transformation;

$$
C \rightarrow C^{(2)}+\frac{B_{K-1}-B_{K-2}}{c_{K-1}^{(2)}-c_{K-2}^{(2)}},
$$

the Eqs.(16a) and (16b) may be rewritten as

$$
\begin{aligned}
& \theta_{n+1}^{(2)}=a_{K}^{(2)} \theta_{n}^{(2)}+c_{K-1}^{(2)} C^{(2)}-B^{(2)}, \\
& \theta_{n+1}^{(2)}=a_{K-1}^{(2)} \theta_{n}^{(2)}+c_{K-2}^{(2)} C^{(2)}-B^{(2)},
\end{aligned}
$$

where 


$$
B^{(2)}=\frac{B_{K-2} c_{K-1}^{K}-B_{K-1} c_{K-2}^{2}}{c_{K-1}^{(2)}-c_{K-2}^{(2)}} .
$$

From Eqs.(16) and (23), it can be seen that Eq.(23a) is of similar form to Eq.(16a) and that Eq.(23b) is of similar form to Eq.(16b).

\section{Concluding Remarks}

Concluding remarks of this paper are summarized as follows;

(i) In the approximate PCM, there exist periodic oscillations for the cut off parameter $C<C_{\mathrm{C}}$, there exist chaotic behavior for the cut off parameter $C>C_{\mathrm{C}}$.

(ii) In the approximate PCM, periodic oscillations $\pi(m)$ exist for $m \leq M_{\max }$.

(iii) The rotation numbers of oscillation in PCM form a part of Farey series and form devil's stairs.

(iv) The order of appearance of the oscillation in PCM has a self similar hierarchy.

\section{References}

[1] Y. Zheng, F. Wang and Y. Fu, Nonlinear Dynamic Stability of Moderately Thick Laminated Plates with Piezoelectric Layers, Int. J. of Nonlinear Sciences \& Numerical Simulation, 10 (4), pp.459-468, 2009.

[2] L. Xin, Modified Projective Synchronization of a New Hyper Chaotic System via Non Linear Control, Commun. Theor. Phys. 52, pp.274-278, 2009.

[3] L. Xin and C. Yong, General Projective Synchronization Between Rössler System and New Unified Chaotic System, Commun. Theor. Phys. 48, pp.132-136, 2007.

[4] J. Liu, J. Lu, Y. Shi, X. Li and Q. Tang, Different Type of Synchronization Phenomena in Unidirectional Coupled Unified Chaotic Systems, Int. J. of Innovative Computing, Information and Control 3, pp.697-708, 2007.

[5] B. Van der Pol, On Oscillation in a Triode Generator with Two Degrees of Freedom, Philos. Mag. 43, pp.700-719, 1927.

[6] M. Cartwright and J.E. Littlewood, On Nonlinear Differential Equation of the Second Order, J. London Math. Soc. 20, pp.180-189, 1945.

[7] E. M. EL-Abbasy, On the Periodic Solution the Van der Pol Oscillator with Large Damping, Proc R. Soc. Edinburgh Sect. A 100, pp.103-106, 1985.
[8] N. Levinson, A Second Order Differential with Singular Solutions, Ann. Math. 50, pp.127-153, 1949.

[9] F. Veerman and F. Verhulst, Quasi periodic phenomena in the Van der Pol-Mathieu equation Department of Mathematics, Utrecht University, pp.1-10, 2009.

[10] J. P. Gollub, T. O. Brunner, and B. G. Danly, Periodicity and Chaos in Coupled Nonlinear Oscillators, Science 200, pp.48-50, 1978.

[11] P. J. Holmes and D. A. Rand, Bifurcation of the Forced Van der Pol Oscillator, Q. Appl. Math. 35, pp.495-509, 1978.

[12] J. Grasman, E. J. M. Veling, and G. M. Willems, Relaxation Oscillation Governed by A Van der Pol Equation with Periodic Forcing Term, SIAM J. Appl. Math. 31, pp.667-676, 1976.

[13] J. Grasman, M. J. W. Jansen, and E. J. M. Veling, Asymptotic methods for relaxation oscillations, North Holland Math. Studies 31, pp.93-111, 1978.

[14] J. Grasman, Relaxation Oscillation of a Van der Pol Equation with Large Critical Forcing Term, Q. App ll. Math. 38, pp.9-15, 1980.

[15] J. Gukenheimer, symbolic Dynamics and Relaxation Oscillations, Physica 1D, pp.227-235, 1980.

[16] U. Parlitz and W. Lauterborn, Period-Doubling and Devil's Staircases of the Driven Van der Pol Oscillator, Phys. Rev. A 36, pp.1428-1434, 1987.

[17] R. Mettin, U. Parlitz and W. Lauterborn, Bifurcation Structure of the Driven Van der Pol Oscillator, Int. J. Bifurcation Chaos 3, pp.1529-1555, 1993.

[18] T. Yamaguchi and H. Ohtagaki, The Order of Appearance of Oscillation Modes of a Piecewise Linear Map, J. Phys. Soc. Jpn. 65, pp.3500-3512, 1996.

[19] T. Yamaguchi and H. Ohtagaki, Oscillation Modes of Forced Nonlinear Systems of Van der Pol Type, J. Phys. Soc. Jpn. 73, pp.588-598, 2004.

[20] A.J. Lichtenburg and M.A. Lieberman, Regular and Chaotic Dynamics, Springer, 1991.

[21] Y.-C. Chen, C.-H. Hsu, M.-J. Wu, T.-H. Chien and S.-Y. Tsai, Nonlinear Resistance Effects on Bifurcations and Chaos Phenomenon of Chua's Circuit, Int. J. of Innovative Computing, Information and Control 2, pp.987-994, 2011.

[22] L. Zhou, Y. Chen and F. Chen, Bifurcation and Chaos of a New 3D Quadratic System, Int. J. of Innovative Computing, Information and Control 4, pp.2481-2488, 2010. 
[23] Y.-C. Chen, C.-H. Hsu, M.-J. Wu, T.-H. Chien and S.-Y. Tsai, Nonlinear Resistance Effects on Bifurcations and Chaos Phenomenon of Chua's Circuit, Int. J. of Innovative Computing, Information and Control 2, pp.987-994, 2011.

[24] F. Zhu, W. Xiang and Z. Gao, Modified Projective Synchronization of Uncertain Chaotic Systems with Input Dead-Zone Nonlinearities, Int. J. of Innovative Computing, Information and Control 5, pp.613-619, 2011.

[25] L. Zhou, Y. Chen and F. Chen, Bifurcation and Chaos of a New 3D Quadratic System, Int. J. of Innovative Computing, Information and Control 4, pp.2481-2488, 2010.

[26] T. Yamaguchi and H. Ohtagaki Stochasticity of Nonlinear Systems of Van der Pol-Mathieu Type, Proc. of 42nd ISCIE Int. Symp. on Stochastic Systems Theory and Its Applications, pp.46-51, 2010. 